\title{
Prognostic markers in resected large cell neuroendocrine carcinoma: a multicentre retrospective analysis
}

\author{
Christian Roesel $^{1 \#}$, Stefan Welter ${ }^{2 \#}$, Karl-Otto Kambartel ${ }^{3}$, Gerhard Weinreich ${ }^{4}$, Thomas Krbek ${ }^{1}$, \\ Monika Serke ${ }^{5}$, Mohammed Ibrahim ${ }^{2}$, Yazan Alnajdawi ${ }^{6}$, Till Plönes $^{6}$, Clemens Aigner ${ }^{6}$ \\ ${ }^{1}$ Department of Thoracic Surgery, Bethanien Hospital, Moers, Germany; ${ }^{2}$ Department of Thoracic Surgery, Lung Hospital, Hemer, Germany; \\ ${ }^{3}$ Department of Pneumology and Allergology, Bethanien Hospital, Moers, Germany; ${ }^{4}$ Department of Pneumology, Ruhrlandklinik, University of \\ Duisburg-Essen, Essen, Germany; ${ }^{5}$ Department of Pneumology, Lung Hospital, Hemer, Germany; ${ }^{6}$ Department of Thoracic Surgery and Thoracic \\ Endoscopy, Ruhrlandklinik, University of Duisburg-Essen, Essen Germany \\ Contributions: (I) Conception and design: All authors; (II) Administrative support: All authors; (III) Provision of study materials or patients: All \\ authors; (IV) Collection and assembly of data: All authors; (V) Data analysis and interpretation: All authors; (VI) Manuscript writing: All authors; (VII) \\ Final approval of manuscript: All authors. \\ \#These authors contributed equally to this work. \\ Correspondence to: Christian Roesel, MD. Department of Thoracic Surgery, Bethanien Krankenhaus, Bethanienstraße 21, 47441 Moers, Germany. \\ Email: Christian.Roesel@bethanienmoers.de.
}

Background: Large cell neuroendocrine carcinomas (LCNEC) are rare pulmonary malignancies. Reported survival rates are heterogeneous and the optimal therapeutic strategy is still debated. The prognosis of LCNEC is generally inferior compared to other non-small lung cancers. In early stages, surgery is recommended but might not be sufficient alone.

Methods: We retrospectively analyzed all consecutive LCNEC patients operated at three institutions with curative intent between May 2005 and January 2017. Data retrieved from individual clinical databases were analyzed with the aim to identify prognostic parameters.

Results: A total of 251 patients with LCNEC underwent curative intent surgery during the observation period. The median age was 64 years, 156 patients $(62.2 \%)$ were male and $88.4 \%$ were smokers. The pathologic AJCC stage was I in 136 patients, II in 77, III in 33, and IV in 5 patients. Median followup was 26 months. Lymphatic vessel invasion $(\mathrm{P}=0.031)$ was identified as significant prognostic factor by multivariable analysis. There was a trend towards decreased survival in patients with blood vessel invasion $(\mathrm{P}=0.067)$. Even in earlier tumor stages, adjuvant chemotherapy had a positive effect on survival. The overall 1 -, 3- and 5-year survival rates were $79.2 \%, 48.6 \%$ and $38.8 \%$ respectively.

Conclusions: Lymphatic invasion (L1) is an independent prognostic factor. Surgery in LCNEC is beneficial in early tumor stages and platinum-based adjuvant chemotherapy may help in achieving better long-term outcomes resulting in most obvious survival differences in stage Ib.

Keywords: Large cell neuroendocrine carcinomas; pulmonary malignancies; lymphatic invasion; curative resection; platinum-based adjuvant chemotherapy

Submitted Sep 21, 2019. Accepted for publication Dec 20, 2019.

doi: $10.21037 /$ jtd.2020.01.07

View this article at: http://dx.doi.org/10.21037/jtd.2020.01.07

\section{Introduction}

Large cell neuroendocrine carcinomas (LCNEC) are a rare subset of lung cancers, accounting for $15 \%$ of neuroendocrine tumors and $2-3 \%$ of all lung cancers (1-2).
The incidence could be even higher since neuroendocrine markers can be missed or misinterpreted if the diagnosis relies on small biopsy specimens (3). About $85 \%$ LCNEC present as a peripheral tumor in contrast to typical and atypical 
carcinoids and small cell lung cancer (SCLC) that are often located centrally (4). Unlike carcinoid tumors, LCNECs often are associated with male sex, older age, and smoking habit (5).

In general, the prognosis in LCNEC patients is poor. A previous study reported that stage IV disease was found in $40 \%$ at initial diagnosis and survival was inferior to other NSCLC tumor types (6). The median overall survival (OS) of stages I-II, III and IV was found to be 32.4, 12.6 and 4.0 months, respectively, in a population-based cancer registry of the Netherlands. Based on retrospective studies the 5 -year survival rates for these patients were between $21 \%$ and $62 \%$ in earlier studies (7-9).

Adjuvant therapy, mainly chemotherapy demonstrated benefits on survival and recurrence $(9,10)$. Although the result was not statistically significant, a retrospective analysis of 144 surgically removed pulmonary LCNEC revealed a better outcome in patients treated with preoperative or postoperative chemotherapy in stage-I, suggesting that the adjuvant therapy has a promising role even in early stage disease (9). Due to the rarity of presentation and persistent difficulty in differential diagnosis, the prognosis and best treatment for this group of malignancies remain uncertain. It is still unclear whether the patients diagnosed with LCNEC should be treated with NSCLC or SCLC-based regimes (11).

Firstly, this triple center retrospective study investigated outcome after surgical resection of LCNEC focusing on prognostic factors that are able to identify subgroups with a worse outcome. A more aggressive treatment strategy could be beneficial for these subgroups. Secondly, we analyzed the value of adjuvant treatment.

\section{Methods}

The data of all consecutive LCNEC patients undergoing curative intended surgery at three different institutions between May 2005 and January 2017 were retrieved from clinical databases and retrospectively analyzed to explore prognostic parameters. Ethical approval was obtained from the ethic committee of the Medical Faculty of the University Duisburg-Essen (13-5363-BO). Follow-up data was obtained from medical records, routine pathology reports and the cancer centers databases. Oncologic staging included computed tomography (CT) of the chest, abdomen, magnetic resonance imaging of the head and bone scintigraphy. Since 2010, standard 18-FDG positron emission tomography (PET) had been integrated into the staging procedure. Preoperative clinical staging and mediastinal staging was performed according to the current guidelines of European Society for Medical Oncology (ESMO) and the European Society of Thoracic Surgeons (ESTS) (12). The treatment decision was guided by an interdisciplinary tumor board in all cases. Patients of the early and late study period were reclassified to the 7 th revision of the TNM classification.

Anatomical resection was completed by systematic lymph node dissection or systematic sampling at the discretion of the surgeon. If mediastinal N2 disease was confirmed by mediastinoscopy or EBUS-TBNA, neoadjuvant chemotherapy or chemo-radiotherapy was administered and surgery was performed in those cases that responded to the induction therapy. All operative specimens were investigated by experienced lung pathologists. Neuroendocrine morphology [abundant mitoses ( $>10$ mitosis $\left./ 2 \mathrm{~mm}^{2}\right)$, neuroendocrine morphology such as rosettes, trabecular growth pattern or palisading of cells and the presence of necroses] and positive staining for CD56, chromogranin A or synaptophysin were applied as criteria for histologic confirmation of LCNEC as recommended by the World Health Organization (13). Lymphatic (L1) and vascular invasion (V1) was certified by the pathologist if direct tumor invasion of lymphatic or vascular structures were observed or if tumor cells were found in these structures.

Depending on co-morbidity, age and cardio-pulmonary function, adjuvant or additive platinum-based chemotherapy was administered in patients with stage I (tumor diameter $>4 \mathrm{~cm}$ ) or stage II and III disease. Adjuvant chemotherapy was administered to selected patients in early stage Ia as well if they were in good clinical condition but presented with $\mathrm{L} 1$ or $\mathrm{V} 1$.

\section{Statistical analysis}

Descriptive variables studied in this research were analyzed and presented in frequency, mean \pm standard deviation and range. Statistical analyses were performed by using Statistical Package for Social Science (SPSS) Version 22.0 (SPSS Inc., Chicago, USA). Survival was defined as the time interval between the date of surgical intervention and the date of death or the last follow-up information of the patients. The latest follow-up of patients was censored.

Kaplan-Meier analysis was performed to compute univariate comparisons between different subsets, including overall survival, $\mathrm{T}$ status, $\mathrm{N}$ status and local lymphatic spread. Differences between survival times of two samples were assessed using log-rank tests. Cox regression analysis was performed in multivariable models. Differences with 
Table 1 Patients demographics and characteristics

\begin{tabular}{|c|c|}
\hline Characteristics & Number of patients (\%) \\
\hline Total & 251 \\
\hline \multicolumn{2}{|l|}{ Gender } \\
\hline Female & $95(37.8)$ \\
\hline Male & $156(62.2)$ \\
\hline \multicolumn{2}{|l|}{ Age (years) } \\
\hline$>65$ years & $112(44.6)$ \\
\hline$\leq 65$ years & $139(55.4)$ \\
\hline Mean (range) & $65(38-87)$ \\
\hline \multicolumn{2}{|l|}{ Smoking habit } \\
\hline Smoker, ex-smoker (>20 py) & $222(88.4)$ \\
\hline Non-Smoker/not known & $29(11.6)$ \\
\hline \multicolumn{2}{|l|}{ Tumor localization } \\
\hline Right upper lobe & $92(36.7)$ \\
\hline Middle lobe & $7(2.8)$ \\
\hline Right lower lobe & $38(15.1)$ \\
\hline Left upper lobe & $73(29.1)$ \\
\hline Left lower lobe & $37(14.7)$ \\
\hline Central, not exactly specifiable & $4(1.6)$ \\
\hline \multicolumn{2}{|l|}{ Lymphangitic invasion } \\
\hline LO & 218 (86.9) \\
\hline L1 & $33(13.1)$ \\
\hline \multicolumn{2}{|l|}{ Vascular invasion } \\
\hline Vo & $188(74.9)$ \\
\hline V1 & $63(25.1)$ \\
\hline \multicolumn{2}{|l|}{ Completeness of resection } \\
\hline Complete (R0) & $243(96.8)$ \\
\hline Incomplete (R1) & $8(3.2)$ \\
\hline \multicolumn{2}{|l|}{ Stage of disease } \\
\hline $\mathrm{IA} / \mathrm{IB}$ & $136(54.2)$ \\
\hline IIA/IIB & $76(30.3)$ \\
\hline IIIA/IIIB & $33(13.1)$ \\
\hline IV & $5(2.0)$ \\
\hline
\end{tabular}

$\mathrm{P}<0.05$ were considered statistically significant. Parameters that probably influenced survival in the univariate analysis with $\mathrm{P}=0.1$ or less were included into a multivariate Cox regression analysis.

\section{Results}

The demographic characteristics of the patients (Table 1) are similar to a previous study (7).

\section{Operative results}

All 251 patients had surgical resections of the primary tumor, which were then histologically categorized as pure (77\%) or mixed (23\%) LCNEC, demonstrating features of both LCNEC and NSCLC, mainly adenocarcinoma. Operative procedures performed included $192(76.5 \%)$ lobectomies, 5 (2\%) bilobectomies, $22(8.8 \%)$ wedge resections, 7 (2.8\%) pneumonectomies, 4 (1.6\%) sleeve lobectomies and $21(8.4 \%)$ anatomical segmentectomies. The number of removed lymph nodes was $12.7 \pm 7.4$. Of the treated patients, 243 patients had a complete resection (97\%) and 8 patients (3\%) had a microscopic residual tumor at the margin (R1) after surgical resection, which was not diagnosed during intraoperative frozen section. There were no intraoperative deaths. One patient developed bronchialstump insufficiency and one other patient developed a fulminant mesenteric ischemia. Both of them died in the further clinical course. Thus, the perioperative 30- and 60 -day mortality rate was $0.8 \%$.

\section{Pathological findings}

An accurate tissue diagnosis by bronchoscopy or CT-guided biopsy was established in 23 (9.2\%) patients preoperatively and was confirmed later on in the resected specimens. All other patients were diagnosed intra- or postoperatively based on the resected specimens.

The pathological examination revealed a median tumor size of $3.5 \mathrm{~cm}$ (mean $3.7 \pm 2.1 \mathrm{~cm}$; $95 \%$ CI, $0.7-13.0 \mathrm{~cm}$ ). The pathologic stage was I in $136(54.2 \%)$, II in 77 (30.7\%), III in $33(13.1 \%)$ and IV in $5(2 \%)$ patients. All stage IV patients presented with an oligometastatic situation. They additionally underwent complete resection of a skin [1], brain [3] or adrenal [1] metastasis. Immunohistochemical staining was positive for CD56 in 214 (85.3\%) patients, Synaptophysin-A in 133 (53\%) patients and Chromogranin-A in 94 (37.5\%) patients. Simultaneous expression of two and three immunohistochemical markers were found in $104(41.4 \%)$ and $45(17.9 \%)$ patients respectively. 
Table 2 Tumor recurrences

\begin{tabular}{lc}
\hline Patient's status & Value \\
\hline Total recurrence, $\mathrm{n}[\%]$ & $118[47]$ \\
Loco-regional recurrence (total), $\mathrm{n}[\%]$ & $33[28]$ \\
Mediastinal & $19[58]$ \\
Pulmonary/pleural metastasis & $14[42]$ \\
Distant recurrence (total), $\mathrm{n}[\%]$ & $85[72]$ \\
Brain & 5 \\
Abdomen & 4 \\
Bone & 6 \\
Multiple (more than two sides) & 70 \\
Median time to local recurrence & $18[1-114]$ months \\
Median time to distant recurrence & $15[1-105]$ months \\
\hline
\end{tabular}

\section{Causes of death}

As mentioned above, $2(0.8 \%)$ patients died in the early postoperative period and one other patient $(0.4 \%)$ died following toxicity of adjuvant chemotherapy and 17 expired of comorbidities during follow-up. 118 additional patients died from tumor relapse. In this subgroup, distant disease occurred in 85 and local recurrence occurred in 33 patients. Local recurrence affected the lung (30.3\%), mediastinum $(57.5 \%)$, pleura $(6.1 \%)$ and the chest wall (6.1\%). A total of 17 (51.5\%) patients with local recurrence received chemotherapy, $8(24.2 \%)$ were treated with radiotherapy and $5(15.2 \%)$ were treated with combined radio-chemotherapy. Two patients were re-operated due to recurrence in the chest-wall. The median time to local recurrence was 18 months. Distant metastases were noted in $85(72 \%)$ cases after a median of 15 months. Among them $70(56.9 \%)$ patients had multiple metastases and were treated with palliative chemotherapy (Table 2).

\section{Survival/prognostic factors}

Survival rate was calculated from the date of lung tumor removal to the last follow-up or date of death. The median follow-up was 26 months (mean $35.5 \pm 31.7$ months). Three patients were lost to follow-up and were excluded from further survival analysis. The 5 -year overall survival for the entire patient cohort was $38.8 \%$. The five-year survival in stages I, II and III was $60.9 \%, 31 \%$ and $22.7 \%$ (Figure $1 A$ ) respectively.
Sex was not associated with survival $(\mathrm{P}=0.295)$. Age $>65$ years was associated with prognosis in the multivariable analysis $(\mathrm{P}=0.017)$ but not significant in the univariate analysis $(\mathrm{P}=0.591)$. There was no survival difference between pure LCNEC and mixed (LCNEC + NSCLC) histology $(\mathrm{P}=0.331)$. The pathologic $\mathrm{T}$-stage (pT) significantly influenced the prognosis. The 5 -year survival of patients with pT1-2 tumors was $43.1 \%$ compared to $23.1 \%$ in patients with a pT3-4 tumors $(\mathrm{P}=0.045)$ (Figure $1 B)$. The 5 -year survival rate decreased from $48.2 \%$ to $26.3 \%$ when lymph node metastases were present $(\mathrm{P}=0.012)$ (Figure $1 C$ ). Patients with lymphatic or vascular invasion faced inferior $(\mathrm{P}=0.007$ and $\mathrm{P}<0.001)$ survival because of higher rates of distant metastasis (Figure 2A,B). Regarding median survival we do not find statistical significance for L0 patients compared with $\mathrm{L} 1$ patients in tumor stages $\mathrm{I}(61 \mathrm{vs}$. 13 months), II (30 vs. 26 months) and III (21 vs. 14 months) (Table 3). Beside stage, lymphatic invasion remained an independent predictor of survival in the multivariable analysis $(\mathrm{P}=0.031)$. Locoregional tumor recurrence was not associated with the presence of $\mathrm{L} 1(\mathrm{P}=0.697)$ or $\mathrm{V} 1$ $(\mathrm{P}=0.974)$.

As a consequence, when survival was analyzed by stage, a worse prognosis was observed in stage III and IV disease compared to stage I-II disease $(\mathrm{P}=0.007)$. Expression of a single neuroendocrine marker (CGA, CD56 or SYN) did not predict overall survival $(\mathrm{P}>0.05)$. There was a trend that the simultaneous expression of two or even three markers was associated with an inferior survival. The median survival was 70 (95\% CI: 40-100) months, 28 (95\% CI: 20 36) months and 26 (95\% CI: 17-35) months in patients with 1,2 and 3 positive neuroendocrine markers $(\mathrm{P}=0.08)$.

\section{Perioperative chemotherapy}

Primary resection was performed in 232 patients (92.4\%), and $19(7.6 \%)$ patients received neoadjuvant chemotherapy or radiochemotherapy. A clinical response to induction therapy was observed in 15 of the 19 patients (78.9\%). A total of $101(40.2 \%)$ surgically resected patients received adjuvant chemotherapy. Data on the chemotherapy regimen were available in 99 of the $120(82.5 \%)$ patients with perioperative chemotherapy. All patients had doublets with cisplatin or carboplatin, one had oxaliplatin. Platin was combined with etoposide, paclitaxel, vinorelbine or pemetrexed in decreasing frequency.

The 5 -years survival was $44.6 \%$ for patients with adjuvant chemotherapy and $37.8 \%$ without $(\mathrm{P}=0.02)$. The 
A

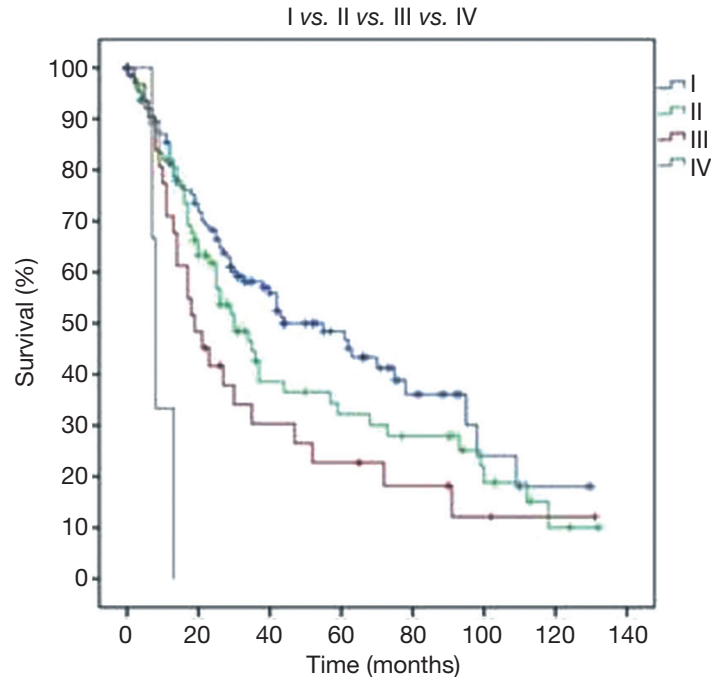

No. at risk

$\begin{array}{ccccccccc}\text { I } & 134 & 84 & 50 & 29 & 13 & 4 & 2 & 0 \\ \text { II } & 76 & 45 & 19 & 15 & 12 & 7 & 2 & 0 \\ \text { III } & 33 & 15 & 8 & 6 & 4 & 2 & 1 & 0 \\ \text { IV } & 4 & 0 & 0 & 0 & 0 & 0 & 0 & 0\end{array}$

C

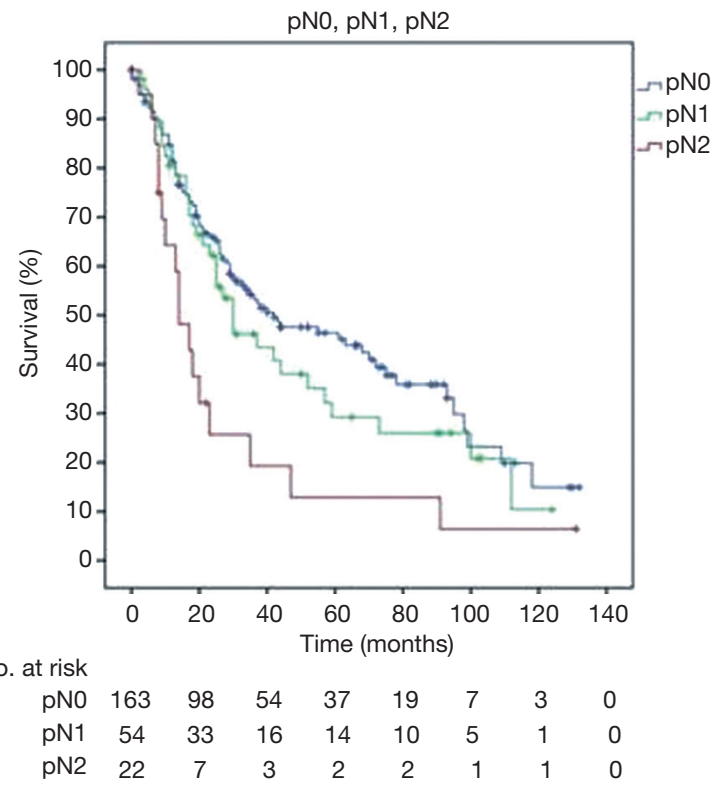

B $\quad$ pT1+2 vs. pT3+4

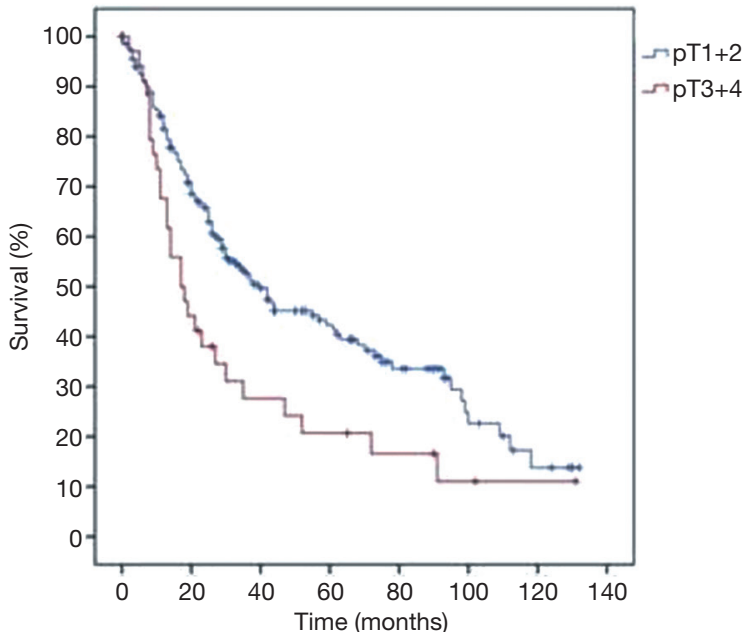

No. at risk

$\begin{array}{lllllllll}\mathrm{pT} 1+2 & 210 & 129 & 69 & 44 & 25 & 11 & 4 & 0\end{array}$

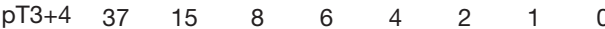

Figure 1 Kaplan-Meier estimates of survival. (A) Stage dependent survival according to Kaplan-Meier overall survival analysis. The number of patients at risc is 247. One case with follow-up data could not be classified by UICC stage. (B) Survival according to pT-stage. T-stage 1 and 2 were grouped together and compared with T-stage 3 and 4 . One case with follow-up data could not be allocated to a T-stage. Survival of patients with T3/T4 tumors was significantly inferior to patients with $\mathrm{T} 1 / \mathrm{T} 2$ tumors ( $\mathrm{P}=0.045$, univariate analysis). (C) Survival according to $\mathrm{pN}$-stage. 8 cases were undefined concerning lymph node involvement $(\mathrm{Nx})$ and one case with follow-up data had no information on $\mathrm{n}$-stage. Therefore 239 patients could be included into survival analysis ( $\mathrm{P}=0.012$, univariate analysis).

administration of perioperative chemotherapy was found as an independent prognostic factor in multivariable analysis. A subgroup analysis of patients in stage I and stage II with and without adjuvant therapy resulted in a positive effect of adjuvant chemotherapy on OS $(\mathrm{P}=0.005$ and $\mathrm{P}=0.0014)$ (Table 3, Figure 3A,B). The effect of adjuvant chemotherapy 
A

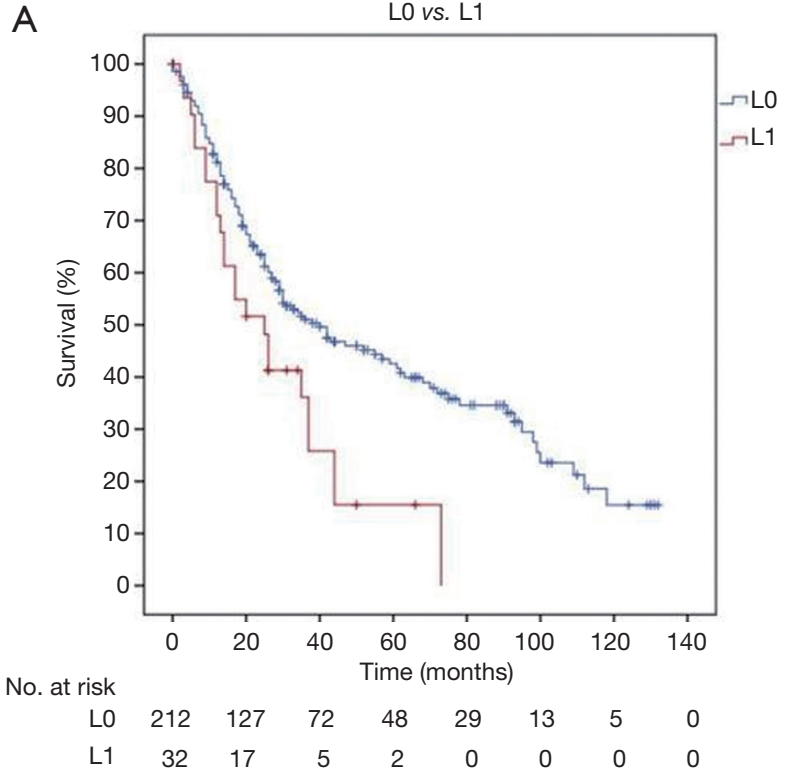

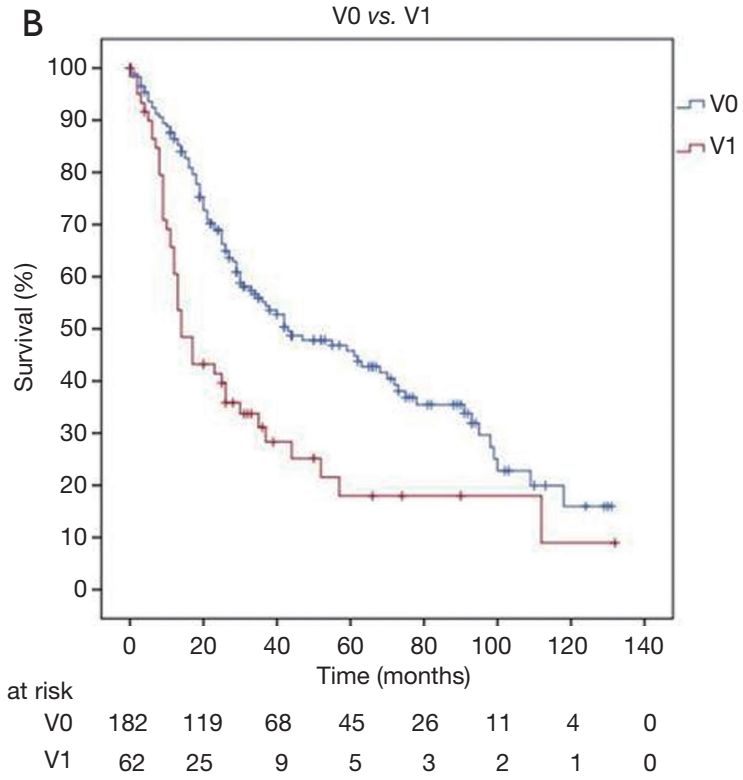

Figure 2 Kaplan-Meier estimates of survival. (A) Overall survival according to the presence or absence of lymphatic invasion ( $\mathrm{P}=0.007$, univariate analysis). Four of 248 cases did not have information about the L-status, therefore 244 cases could be included into survival analysis. (B) Overall survival according to the presence or absence of vascular invasion. 244 cases could be analyzed (A). V1 was associated with inferior prognosis in the univariate analysis $(\mathrm{P}<0.001)$.

was analyzed in the subgroups with different marker expression profiles. No difference was found for patients with CD56 or Synaptophysin, whereas patients positive for CgA had inferior median survival after adjuvant chemo compared to $\mathrm{CgA}$ negative patients (99 vs. 25 months, $\mathrm{P}=0.003)$. To define the effect of adjuvant chemo in early tumor stages IA and IB more precisely, we included age, sex, ECOG score and histology (LCNEC $v s$. mixed tumors) into a Cox regression model. Adjuvant chemo still had a significant positive effect on survival with a HR of 0.379 (95\% CI: 0.168-0.852, $\mathrm{P}=0.019$ ).

\section{Discussion}

In this study, we present the result of a large contemporary multi-institutional series of 251 resected patients with LCNECs of the lung, focusing on clinicopathologic factors, effects of perioperative chemotherapy and survival outcomes. The study aimed at determining prognostic factors, identifying patients with a poorer prognosis, who might benefit from a more aggressive therapy. In contrast to other neuroendocrine lung tumors, we found that most $(85 \%)$ LCNEC had grown in the periphery of the lung. This might be one of the reasons why most of the patients were asymptomatic and the tumor was diagnosed during routine imaging. In addition, this might also be an explanation why early tumor stages were over-represented in our retrospective data sets. Tumor stage and T-stage was associated with prognosis in our study as was described by others as well (14). Among others, lymphatic and vascular invasion was identified in univariable analysis, however lymphatic invasion remained an independent prognostic factor in multivariable analysis. Those cases are correlated with inferior prognosis after resection of LCNEC.

Previous studies reported that the presence of vascular invasion or lymphatic invasion was a significantly unfavorable prognostic factor in many malignant tumors (15). Lymphatic or vascular invasion (L1, V1) triggers an important process in the progression and local spread of cancer cells. Lymphatic vessels are regarded as an important route by which neoplastic cells reach local lymph nodes (16). When tumor cells penetrate a blood or lymphatic vessel, they can detach, disseminate and arrest in the microvasculature and migrate into the organs tissue. The presence of vascular invasion by neoplastic cells might indicate that the cancers are in a metastatic phase. In a meta-analysis, Saijo et al. reported that L1 is a prognostic factor for survival in patients with NSCLC (17). However, 
Table 3 Prognostic factors and their association with survival

\begin{tabular}{|c|c|c|c|}
\hline Factor & $\begin{array}{c}\text { Univariable } \\
\text { P }\end{array}$ & \multicolumn{2}{|c|}{ Multivariable } \\
\hline \multicolumn{4}{|l|}{ Age } \\
\hline$<65$ vs. $\geq 65$ years & 0.591 & $1.029(1.005-1.053)$ & 0.017 \\
\hline \multicolumn{4}{|l|}{ Sex } \\
\hline \multicolumn{4}{|l|}{ T-stage } \\
\hline pT1/2 vs. pT3/4 & 0.045 & $1.482(0.842-2.609)$ & 0.172 \\
\hline \multicolumn{4}{|l|}{$\mathrm{N}$-stage } \\
\hline N0 vs. N1 vs. N2 & 0.012 & & \\
\hline \multicolumn{4}{|l|}{ L-stage } \\
\hline L0 vs. L1 stage I & 0.110 & & \\
\hline L0 vs. L1 stage II & 0.184 & & \\
\hline L0 vs. L1 stage III & 0.332 & & \\
\hline \multicolumn{4}{|l|}{ V-stage } \\
\hline V0 vs. V1 & 0.001 & $1.612(0.967-2.690)$ & 0.067 \\
\hline \multicolumn{4}{|l|}{ Completeness of resection* } \\
\hline $\mathrm{R} 0$ vs. R1/R2 & 0.002 & * & * \\
\hline \multicolumn{4}{|l|}{ Chemotherapy } \\
\hline Stage I & 0.005 & & \\
\hline Stage II & 0.014 & & \\
\hline Chromogranin A pos. vs. neg. & 0.003 & & \\
\hline CD56 pos. vs. neg. & 0.983 & & \\
\hline Synaptophysin pos. vs. neg. & 0.743 & & \\
\hline \multicolumn{4}{|l|}{ Presence of tumor locoregional relapse } \\
\hline Relapse vs. no relapse & 0.042 & $1.619(0.928-2.825)$ & 0.090 \\
\hline \multicolumn{4}{|l|}{ Immunohistochemical marker } \\
\hline Chromogranin A & 0.132 & & \\
\hline Synaptophysin & 0.406 & & \\
\hline CD56 & 0.584 & & \\
\hline 1 vs. 2 vs. 3 marker & 0.078 & & \\
\hline
\end{tabular}

${ }^{*}$, resection status was not included into multivariable analysis as only 2 of 7 cases had complete data of all other parameters. 

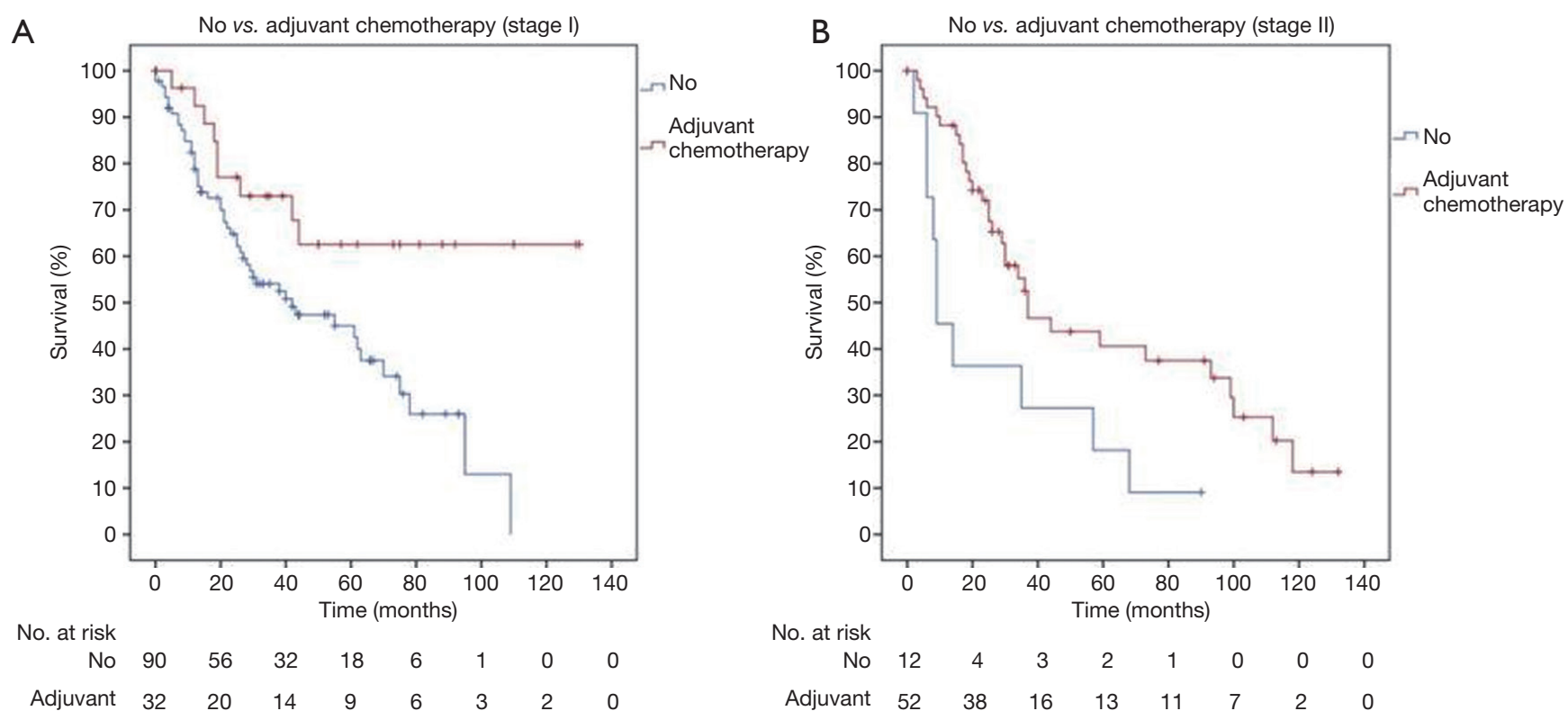

Figure 3 Kaplan-Meier estimates of survival. (A) Survival according to adjuvant chemotherapy in stage I patients (P=0.005). Of 125 patients in stage I, 2 were lost to follow-up and adjuvant treatment was unclear in 1 patient. Therefore 122 cases could be included into further survival analysis. (B) Survival according to adjuvant chemotherapy in stage II patients. Of 76 patients in stage II, 6 patients had been given neoadjuvant treatment and there was a lack of information about adjuvant treatment in another 6 cases. Therefore 64 cases could be included into further survival analysis. Univariate analysis demonstrating a positive effect of adjuvant chemotherapy in stage $\mathrm{I}$ and stage $\mathrm{II}$ ( $\mathrm{P}=0.005$ and 0.014).

L1 and V1 as prognostic factors for survival in NSCLC remain controversial and they are not considered in the current tumor staging system. The factor V1 has been reported as a strong predictor of recurrence or the cause of death among cancer patients by others, which was independent of lymph node metastasis (18).

The clinicopathologic features in this study were comparable with other studies. LCNECs affected males $(62 \%)$ and almost exclusively smokers $(>88 \%)$ and present the same clinical and radiological features like other lung cancers. Therefore, as of now, it is impossible to distinguish LCNEC from other NSCLC by clinical parameters. LCNEC's are considered as a separate entity from other neuroendocrine tumors for clinical characteristics, prognosis, and survival. Varlotto et al. reported that the clinical, histopathological and biological characteristics of LCNEC were more similar to large-cell carcinoma than to small cell lung cancer (SCLC) (11). But histological differentiation between LCNEC and SCLC can be very challenging as both tumor entities often share many common features. Since 2004 the World Health Organization (WHO) categorizes LCNEC in the group of NSCLC.
Regarding immunohistochemistry analysis, pulmonary LCNECs express typical neuroendocrine markers such as chromogranin A, CD56 and synaptophysin, which are necessary to define the diagnosis. As reported in a previous study (4), we could establish preoperative LCNEC diagnosis only in $9.2 \%$. The low rate might be explained by the fact that most nodules were localized in the periphery and intraoperative frozen section analysis was the standard procedure to establish histological diagnosis in our units. This rate was even higher than the previously published $(4.8 \%)$ results (4). We now consider that this observed increment can be better explained by a growing awareness among pathologists, greater sample size and sample preparation during bronchoscopy and a more standardized and frequent immunohistochemical staining for neuroendocrine markers at routine pathological work-up. Neuroendocrine marker profiles may predict prognosis and therapy concepts. In our study we found a trend that the presence of more than one neuroendocrine marker was associated with inferior prognosis $(\mathrm{P}=0.08)$. In the study by Tanaka et al. perioperative chemotherapy resulted in better overall survival than surgery alone $(\mathrm{P}=0.042)$. Interestingly, in the non-triple-positive group, 
a significantly higher 5-year survival rate was observed for the patients who underwent perioperative chemotherapy than in those who underwent surgery alone $(\mathrm{P}=0.008)$. No difference was found in the triple-positive group (19). We observed a reduced effect of adjuvant chemotherapy on OS when $\mathrm{CgA}$ was positive. The triple-positive group was too small to perform separate survival analysis. At least one can state, that CgA in the Tanakas (19) paper was positive in the triple-positive group and hence associated with inferior survival. This might be the key for future study conceptions.

\section{Therapy}

Primary surgery should be the first option in operable patients with stages I and II. This approach offers also a way to obtain an accurate diagnosis (8-10). Unfortunately, the majority $(70 \%)$ of pulmonary LCNEC cases are not eligible for surgical resection due to the high prevalence of metastases at initial diagnosis. Even those patients with LCNEC at stage I have a poor prognosis, with 5 -year survival rates ranging from $27 \%$ to $67 \%$ (10). Therefore, surgery alone may not be sufficient. As we did in our study, the retrospective analysis of 144 surgically removed pulmonary LCNECs by Veronesi revealed a better outcome, with preoperative or postoperative chemotherapy in stage I disease although not statistically significant. They stated that adjuvant therapy has a promising role in earliest diagnosed disease (9). Iyoda et al. found a significantly better 5 -year OS in those patients treated with complete resection and adjuvant cisplatin-etoposide chemotherapy compared to those having no adjuvant treatment (20). Rossi et al. reported that adjuvant chemotherapy based on cisplatin plus etoposide was effective for patients with LCNEC (6). Saji et al. retrospectively enrolled 45 patients with surgically resected LCNEC in a study (21). Twentythree patients received preoperative or postoperative chemotherapy and their 5 -years survival was superior (87.5\%) compared with the group without $(58.5 \%)(\mathrm{P}=0.04)$. In our study patients with stage I disease benefited from adjuvant chemotherapy. We had the impression, that even stage IA patients would benefit. Unfortunately, the survival advantage in stage IA with chemo was not significant $(\mathrm{P}=0.472)$. As the sample size of stage IA patients with adjuvant therapy was lower than in stage IB it cannot be excluded that a type II error may have occurred. The studies of Tanaka et al. (19) and Filosso et al. (10) also presented that perioperative chemotherapy might be beneficial for the survival of patients with pulmonary
LCNEC. These facts underline the importance of adjuvant treatment in LCNEC regarding over-all survival. New therapy options such as biological and molecular targeted therapies or immunotherapies have been used in individual cases with promising effects $(22,23)$.

\section{Survival}

Pulmonary LCNEC behave aggressively. The 5-year survival in stages I, II and III was $60.9 \%, 31 \%$ and $22.7 \%$ respectively in our study. In this study we found that lymph vessel invasion and blood vessel invasion are important prognosticators. The prevalence of $\mathrm{L} 1$ was $13.1 \%$ and much lower than reported in a meta-analysis by Wang et al. (15). Blood vessel invasion was $25.1 \%$ in our series and comparable with other reported NSCLC series 29.8\% (17). Both, L1 and V1 were associated with impaired survival in other NSCLC as well $(20,21)$. Furthermore, L1 was associated with increased numbers of local recurrence after NSCLC resection (15). A significant number of local recurrences were found in patients with V1 in NSCLC stage I after resection (17). Increased local recurrences with L1 $(\mathrm{P}=0.697)$ or V1 $(\mathrm{P}=0.974)$ were not found in our study. In summary, we could demonstrate impaired survival in LCNEC tumors with L1 and V1. The prognostic impact of L1 was evaluated earlier for other NSCLC patients in a large meta-analysis as well (17). Due to the retrospective design of the study we did not perform a sample size calculation. Therefore, we cannot exclude that a type II error, including the prognostic importance of L1, may have occurred

\section{Limitations}

This study has some limitations due to the retrospective nature of the study and the long observation period including changes of patient management (e.g., PET-CT). Furthermore, the LCNECs are sometimes components of heterogenous tumors so that some cases might have been missed during normal pathologic work-up, especially in larger tumors. L1 was reported when obviously present at routine specimen work-up. A prospective focus on L1 might have identified further cases. On the other hand, the results of this large data set were very similar in all centers and comparable with published evidence. Because we did not differentiate between race and most patients but not all were Caucasians, our results should be interpreted with care in other populations. 


\section{Conclusions}

This study analyzed a large pooled dataset and reported clinicopathologic factors and survival outcomes in patients with LCNEC. Besides stage, lymphatic invasion (L1) is an important prognostic factor for impaired survival after surgery. Although radical surgery is the mainstay of curative treatment and adjuvant chemotherapy is standard in stage IIIA, adjuvant therapy should be considered in stages IB-IIB, but may be ineffective when $\mathrm{CgA}$ is present in the tumor. A prospective randomized clinical trial is recommended to elucidate the effect of adjuvant therapy in early stages. Unfavourable prognostic factors like L1 might be also considered for inclusion in the future revision of the TNM staging system and for therapy decisions concerning adjuvant chemotherapy.

\section{Acknowledgments}

Funding: None.

\section{Footnote}

Conflicts of Interest: SW serves as the unpaid editorial board member of Journal of Thoracic Disease from May 2019 to Apr 2021. The other authors have no conflicts of interest to declare.

Ethical Statement: The authors are accountable for all aspects of the work in ensuring that questions related to the accuracy or integrity of any part of the work are appropriately investigated and resolved. Ethical approval was obtained from the ethic committee of the Medical Faculty of the University Duisburg-Essen (13-5363-BO).

Open Access Statement: This is an Open Access article distributed in accordance with the Creative Commons Attribution-NonCommercial-NoDerivs 4.0 International License (CC BY-NC-ND 4.0), which permits the noncommercial replication and distribution of the article with the strict proviso that no changes or edits are made and the original work is properly cited (including links to both the formal publication through the relevant DOI and the license). See: https://creativecommons.org/licenses/by-nc-nd/4.0/.

\section{References}

1. Travis WD, Linnoila RI, Tsokos MG, et al.
Neuroendocrine tumors of the lung with proposed criteria for large-cell neuroendocrine carcinoma. An ultrastructural, immunohistochemical, and flow cytometric study of 35 cases. Am J Surg Pathol 1991;15:529-53.

2. Fasano M, Della Corte CM, Papaccio F, et al. Pulmonary large-cell neuroendocrine carcinoma: from epidemiology to therapy. J Thorac Oncol 2015;10:1133-41.

3. Kyritsis I, Krebs B, Kampe S, et al. Erroneous diagnosis of small cell lung cancer based on small biopsies with farreaching consequences: case report of a typical carcinoid tumor. J Thorac Dis 2017;9:E99-102.

4. Roesel C, Terjung S, Weinreich G, et al. A SingleInstitution Analysis of the Surgical Management of Pulmonary Large Cell Neuroendocrine Carcinomas. Ann Thorac Surg 2016;101:1909-14.

5. Sánchez de Cos Escuín J. Diagnosis and treatment of neuroendocrine lung tumors. Arch Bronconeumol 2014;50:392-6.

6. Rossi G, Cavazza A, Marchioni A, et al. Role of chemotherapy and the receptor tyrosine kinases KIT, PDGFRalpha, PDGFRbeta, and Met in large-cell neuroendocrine carcinoma of the lung. J Clin Oncol 2005;23:8774-85.

7. Travis WD, Rush W, Flieder DB, et al. Survival analysis of 200 pulmonary neuroendocrine tumors with clarification of criteria for atypical carcinoid and its separation from typical carcinoid. Am J Surg Pathol 1998;22:934-44.

8. Zacharias J, Nicholson AG, Ladas GP, et al. Large cell neuroendocrine carcinoma and large cell carcinomas with neuroendocrine morphology of the lung: prognosis after complete resection and systematic nodal dissection. Ann Thorac Surg 2003;75:348-52.

9. Veronesi G, Morandi U, Alloisio M, et al. Large cell neuroendocrine carcinoma of the lung: a retrospective analysis of 144 surgical cases. Lung Cancer 2006;53:111-5.

10. Filosso PL, Guerrera F, Evangelista A, et al. ESTS Lung Neuroendocrine Working Group Contributors. Adjuvant chemotherapy for large-cell neuroendocrine lung carcinoma: results from the European Society for Thoracic Surgeons Lung Neuroendocrine Tumours Retrospective Database. Eur J Cardiothorac Surg 2017;52:339-45.

11. Varlotto JM, Medford-Davis LN, Recht A, et al. Should large cell neuroendocrine lung carcinoma be classified and treated as a small cell lung cancer or with other large cell carcinomas? J Thorac Oncol 2011;6:1050-8.

12. Vansteenkiste J, Crinò L, Dooms C, et al. 2nd ESMO Consensus Conference on Lung Cancer: early-stage nonsmall-cell lung cancer consensus on diagnosis, treatment 
and follow-up. Ann Oncol 2014;25:1462-74.

13. Travis WD, Brambilla E, Noguchi M, et al. International Association for the Study of Lung Cancer: American Thoracic Society/European Respiratory Society international multidisciplinary classification of lung adenocarcinoma. J Thorac Oncol 2011;6:244-85.

14. Cattoni M, Vallières E, Brown LM, et al. Large Cell Neuroendocrine Tumor Size $>3 \mathrm{~cm}$ Negatively Impacts Long-Term Outcomes after R0 Resection. World J Surg 2019;43:1712-20.

15. Wang J, Wang B, Zhao W, et al. Clinical significance and role of lymphatic vessel invasion as a major prognostic implication in non-small cell lung cancer: a meta-analysis. PLoS One 2012;7:e52704.

16. Rouzaut A, Irigoyen M, Montuenga LM. Lymphangiogenesis and lung cancer. J Thorac Oncol 2007;2:384-6.

17. Saijo T, Ishii G, Ochiai A, et al. Evaluation of extratumoral lymphatic permeation in non-small cell lung cancer as a means of predicting outcome. Lung Cancer 2007;55:61-6.

18. Wang J, Chen J, Chen X, et al. Blood vessel invasion as a strong independent prognostic indicator in non-small cell lung cancer: a systematic review and meta-analysis. PLoS One 2011;6:e28844.

Cite this article as: Roesel C, Welter S, Kambartel KO, Weinreich G, Krbek T, Serke M, Ibrahim M, Alnajdawi Y, Plönes T, Aigner C. Prognostic markers in resected large cell neuroendocrine carcinoma: a multicentre retrospective analysis. J Thorac Dis 2020;12(3):466-476. doi: 10.21037/jtd.2020.01.07
19. Tanaka Y, Ogawa H, Uchino K, et al. Immunohistochemical studies of pulmonary large cell neuroendocrine carcinoma: a possible association between staining patterns with neuroendocrine markers and tumor response to chemotherapy. J Thorac Cardiovasc Surg 2013;145:839-46.

20. Iyoda A, Hiroshima K, Moriya Y, et al. Postoperative recurrence and the role of adjuvant chemotherapy in patients with pulmonary large-cell neuroendocrine carcinoma. J Thorac Cardiovasc Surg 2009;138:446-53.

21. Saji H, Tsuboi M, Matsubayashi J, et al. Clinical response of large cell neuroendocrine carcinoma of the lung to perioperative adjuvant chemotherapy. Anticancer Drugs 2010;21:89-93.

22. Filosso PL, Ruffini E, Oliaro A, et al. Large-cell neuroendocrine carcinoma of the lung: a clinicopathologic study of eighteen cases and the efficacy of adjuvant treatment with octreotide. J Thorac Cardiovasc Surg 2005;129:819-24.

23. De Pas TM, Giovannini M, Manzotti M, et al. Largecell neuroendocrine carcinoma of the lung harboring EGFR mutation and responding to gefitinib. J Clin Oncol 2011;29:e819-22. 